\title{
Polariton mediated resonance energy transfer in a fluid
}

Cite as: J. Chem. Phys. 153, 034111 (2020); https://doi.org/10.1063/5.0011562

Submitted: 22 April 2020 . Accepted: 23 June 2020 . Published Online: 17 July 2020

Dale Green (D), Garth A. Jones (D), and A. Salam (D)

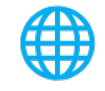

\section{ARTICLES YOU MAY BE INTERESTED IN}

Molecular polaritons for controlling chemistry with quantum optics

The Journal of Chemical Physics 152, 100902 (2020); https://doi.org/10.1063/1.5136320

Photoisomerization-coupled electron transfer

The Journal of Chemical Physics 153, 034301 (2020); https://doi.org/10.1063/5.0013468

Polaritonic normal modes in transition state theory

The Journal of Chemical Physics 152, 161101 (2020); https://doi.org/10.1063/5.0007547

\section{Lock-in Amplifiers up to $600 \mathrm{MHz}$}
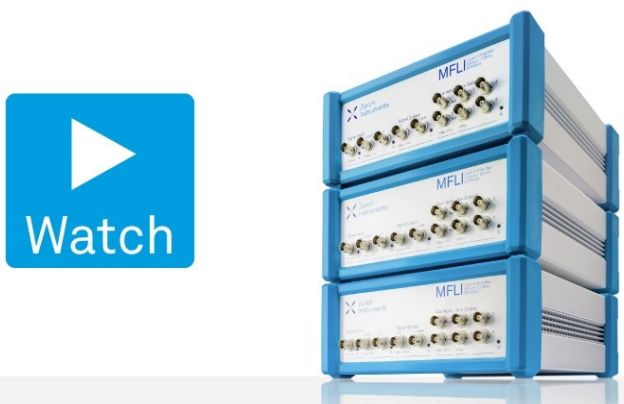

J. Chem. Phys. 153, 034111 (2020); https://doi.org/10.1063/5.0011562 


\title{
Polariton mediated resonance energy transfer in a fluid
}

\author{
Cite as: J. Chem. Phys. 153, 034111 (2020); doi: 10.1063/5.0011562 \\ Submitted: 22 April 2020 - Accepted: 23 June 2020 • \\ Published Online: 17 July 2020
}

Dale Green, (D) Garth A. Jones, ${ }^{1, a)}$ (D) and A. Salam ${ }^{2, a)}$ (D)

\author{
AFFILIATIONS \\ 'School of Chemistry, University of East Anglia, Norwich NR4 7TJ, United Kingdom \\ ${ }^{2}$ Department of Chemistry, Wake Forest University, Winston-Salem, North Carolina 27109, USA
}

a) Authors to whom correspondence should be addressed: garth.jones@uea.ac.uk and salama@wfu.edu

\begin{abstract}
The focus of this work is on a microscopic quantum electrodynamical understanding of cumulative quantum effects in resonance energy transfer occurring in an isotropic and disordered medium. In particular, we consider quantum coherence, defined in terms of interferences between Feynman pathways, and analyze pure-amplitude and phase cross terms that appear in the Fermi golden rule rate equation that results from squaring the matrix element for mediated energy transfer. It is shown that pure-amplitude terms dominate in the near-zone when chromophores are close in proximity to one another (within a few nanometers), and phase cross terms dominate toward the far-zone when phase differences between different Feynman pathways begin to emerge. This can be understood in terms of physical attributes of the mediating photon, whose character becomes more real at long distances, coinciding with vanishing longitudinal components of the field, as transverse components begin to dominate.
\end{abstract}

Published under license by AIP Publishing. https://doi.org/10.1063/5.0011562

\section{INTRODUCTION}

Recently, we presented a microscopic quantum electrodynamical (QED) theory describing damping and decoherence in condensed phase resonance energy transfer (RET). Whereas in open system density matrix approaches, coherences are typically defined as complex off-diagonal elements, ${ }^{2}$ in our treatment, coherence arises from interference effects between Feynman pathways connecting the initial state to the final state. Consequently, damping in RET systems can be understood to take two physical manifestations: (i) energy conserving phase damping, which occurs as a result of geometric and dispersive distortions within the system, and (ii) dissipative amplitude damping in which the virtual photons mediating the RET process are lost to the environment through alternative quantum channels, namely, loss due to vibrational damping and absorption through a material medium with a complex refractive index. Both of these result in a rapid quantum decoherence in the condensed phase. Full details are described in Ref. 1.

An important aspect of decoherence in RET that emerges from this microscopic picture is the central role of the molecular polarizability. This quantity dictates how mediating virtual photons are scattered from intervening chromophores situated between or in the vicinity of the donor, $\mathrm{D}$, and the acceptor, A. The scattering of photons by passive chromophores can significantly enhance the rate of RET, but the polarizability is also responsible for amplitude damping at the molecular centers. It is well known that decoherence occurs much more rapidly in a more disordered environment. Our focus is on understanding how quantum coherence occurs via randomly oriented mediating chromophores, as would be the case of RET in an isotropic fluid. This can be understood in terms of the efficiency with which mediators scatter virtual photons.

Rigorously accounting for the effects of a bath on inter-particle interactions, such as those occurring in RET, is a challenging undertaking. At its heart RET, such as other forms of coupling between material particles, is a manifestation of the Coulomb interaction between the charged particles associated with each center. To accurately treat RET in a medium, a number of different couplings need to be accounted for. These include the very process by which energy migrates from $\mathrm{D}$ to $\mathrm{A}$, the interactions amongst the particles comprising the bath if the environment is described microscopically, and the coupling between these two subsystems. This 
leads to different possibilities for the introduction of the refractive index, depending on how the medium is to be described, and the role played by the chromophores. ${ }^{3,4}$ Factors such as the fluorescence and absorption rates, the distance and orientational dependence of the transition dipole moments, and the properties of the bath (local field and screening corrections) will all affect the strength of the coupling between the pair exchanging energy and, consequently, modify the rate. A common macroscopic approach to deal with the surrounding environment, widely employed in quantum chemical computations, is to place the quantum system of interest in a cavity of finite volume, which in turn resides in a medium with a prescribed frequency dependent dielectric constant. A variety of approaches have been developed, ranging from the primitive Onsager dipole model to more sophisticated polarizable continuum methods, to describe the change in the polarization characteristics of one or more particles and their surroundings. Together, these come under the general banner of self-consistent reaction field methods. ${ }^{5,6}$

Microscopic treatments of the bath, in which its particulate character is manifest, have previously been considered. One fundamental attempt involved describing the electromagnetic field and the atoms or molecules making up the environment as constituting the reservoir, which is quantized. ${ }^{7,8}$ Initially, the particles of the medium were taken to be two-level species, but in later work, this was extended to realistic many-level systems. A quantum mechanical particle is embedded in such a bath and may undergo absorption or emission of light, or couple to other atoms or molecules not comprising the medium. An early application of this construct to an elementary interaction occurred with RET. ${ }^{8-12}$ In vacuum, the acquisition of electronic and/or vibrational excitation by an acceptor moiety initially in its ground state from an excited donor molecule is well understood, thanks to a number of $\mathrm{QED}^{13-17}$ calculations that have been performed over the years of the probability for the migration of energy between the pair. ${ }^{18-31}$ We build on this framework by accounting for the complex refractive index of the medium, whose real part gives rise to dispersion and whose imaginary part describes absorption, with the latter commonly represented by the Beer-Lambert law. It is also worth mentioning recent efforts by $\mathrm{Hsu}$ and co-workers, ${ }^{32-34}$ who employed macroscopic QED to treat RET in an inhomogeneous absorbing and dispersive medium whose relative permeability is one, with a particular focus on plasmonic structures. Accurate electromagnetic field amplitudes are computed using finite difference time domain methods, while material properties are obtained via time-dependent density functional theory (TD-DFT). In combination, these led to time domain electrodynamics being applied to RET (TED-RET).

As a starting point, we briefly re-consider the transfer of excitation energy arising from the exchange of a single virtual photon between transmitter and receiver entities in the absence of a medium. For a freely tumbling donor-acceptor system, the Fermi golden rule migration rate is given $b^{14}$

$$
\langle\Gamma\rangle=\frac{\eta}{36 \pi \varepsilon_{0}^{2} \hbar \rho^{6}}\left|\vec{\mu}^{0 m}(D)\right|^{2}\left|\vec{\mu}^{m 0}(A)\right|^{2}\left[k_{m 0}^{4} \rho^{4}+k_{m 0}^{2} \rho^{2}+3\right],
$$

with the angular brackets surrounding $\Gamma$, denoting that a rotational average has taken place, as would be applicable for molecules in a solution or, more generally, in the fluid phase. $\vec{\rho}=\vec{R}_{A}-\vec{R}_{D}$ is the distance between the donor and the acceptor, which is beyond the orbital overlap for D and A positioned at $\vec{R}_{D}$ and $\vec{R}_{A}$, respectively. The transition electric dipole moments are given by $\vec{\mu}^{0 m}(\xi)$ $=\left\langle 0^{\xi}|\vec{\mu}(\xi)| m^{\xi}\right\rangle$, for $\xi=\mathrm{D}$, A. For decay of excitation associated with the downward transition occurring in the emitter, the energy is given in wavenumber units as $k_{m 0}=\left(E_{m}-E_{0}\right) / \hbar c$, with the density of states being $\eta$.

Equation (1) is a consequence of the unified theory of RET. $^{21,25,28,29}$ It encompasses the inverse sixth power dependence of the rate on inter-particle separation distance well known from Förster theory, and it also includes the inverse square behavior indicative of exchange of energy via a radiative mechanism. Each functional form may be obtained from Eq. (1) on taking near- and far-zone asymptotic limits, respectively. Furthermore, the intermediate-zone represented by the $\rho^{-4}$ term needs to be included when $\rho \sim \lambda / 2 \pi$.

The introduction of the dielectric medium, which is characterized by a complex refractive index $n$, turns free-space virtual photons into bath-dressed virtual photons or polaritons, and the rate formula [Eq. (1)] is modified as a result (see Sec. II and Refs. 8, 10, and 12). Terms dependent upon $\rho^{-3}$ and $\rho^{-5}$ appear in addition to the near-, intermediate-, and far-zone terms, as well as contributions proportional to $\operatorname{Re}(n)$ and/or $\operatorname{Im}(n)$. Screening and local field factors are also contained in the expression for the transfer rate. Furthermore, because the surroundings may be absorbing, the correct exponential decay factor proportional to $\operatorname{Im}(n)$ scales the rate formula.

In previous work, ${ }^{1}$ we considered the influence of a polarizable third molecule, $\mathrm{T}$, on modifying the resonant exchange of energy between $\mathrm{D}$ and $\mathrm{A}$, in a medium, when all three chromophores are maintained in a fixed geometry, as may be the case for a crystal lattice, for example. The focus of that study was on understanding to what extent RET could be enhanced by quantum coherence, defined as arising from interferences between Feynman pathways. Furthermore, we showed that there are distinctly different decoherence mechanisms, some involving energy conserving (phase) damping and others due to dissipative (amplitude) damping. Other studies have also considered the effects of a medium on pair and thirdbody mediated RET, but without considering the explicit role of damping in a quantitative way. ${ }^{34-39}$ We note that there are a number of other processes in which energy is transferred amongst three particles which have also previously been examined, in addition to the prototypical transfer between $\mathrm{D}, \mathrm{A}$, and $\mathrm{T}$ in a medium. ${ }^{40-43}$ Some of these include energy pooling schemes such as twin-donor energy migration, cooperative and accretive mechanisms that facilitate upconversion, downconversion, and sensitization in rare-earth doped systems, as well as cavity enhanced three-body upconversion. ${ }^{44-48}$ A few publications have even dealt with RET involving four bodies. ${ }^{49-5}$

In this work, we seek to understand the relative contribution of pure-amplitude and phase interference cross terms to the overall rate of RET in an isotropic and disordered fluid. It is well known that quantum decoherence occurs rapidly in liquid solutions; however, several years ago, there were a number of surprising experimental results indicating that coherences of quantum origin may occur in photosynthetic systems. ${ }^{53-55}$ Although not fluids, these biological systems are thought to be highly mobile with 
significant levels of disorder in their native states at ambient temperatures. However, we note that the nature of these coherences is now thought to be "emergent," rather than genuine quantum coherences. ${ }^{56}$ Nevertheless, genuine quantum coherence in molecular systems can be maintained in cavities that are free from significant environmental noise. ${ }^{57,58}$ Consequently, in this work, we also seek to understand spatial distances over which pureamplitude and phase interference cross terms may dominate. This is carried out by examining such effects occurring in an isotropic system involving third-body mediated RET over a range of separation distances between the chromophores. As detailed below, rotational averaging is used to calculate analytical expressions for rates of RET, and computer simulations are used to quantify the relative contributions of different coherence effects to the overall rate.

The paper is organized as follows: Sec. II briefly describes pair RET in a medium occurring via polariton exchange, before the third body is introduced and the QED theory is developed, and the total transfer rate arising from direct and all indirect pathways is evaluated. In Secs. III and IV, we derive analytical results for thirdbody mediated RET in a fluid, and finally, we model the analytical solutions computationally in Sec. V, before concluding and looking forward in Sec. VI.

\section{PAIR RET IN A FLUID}

Before considering the effect of a third body in mediating the transfer rate in a medium using the polariton model, we briefly reexamine the change in the form of the matrix element and Fermi golden rule rate when a bath-dressed photon is exchanged between a donor, $\mathrm{D}$, and an acceptor, $\mathrm{A}$. The chromophores are taken to be chemically identical, although the theory can be easily extended to energetically non-degenerate systems. With the donor initially in the excited electronic state $\left|m^{D}\right\rangle$ and the acceptor in the ground state $\left|0^{A}\right\rangle$, the initial state for the total system, in which there are no polariton modes occupied, is

$$
|i\rangle=\left|m^{D}, 0^{A}\right\rangle .
$$

On de-excitation, $\mathrm{D}$ ends up in its lowest level $\left|0^{D}\right\rangle$, while after transfer of energy, $\mathrm{A}$ is excited to state $\left|m^{A}\right\rangle$, with the total final state expressed as

$$
|f\rangle=\left|0^{D}, m^{A}\right\rangle .
$$

For a system comprising two molecules, the radiation field, the interaction between them, and the total QED Hamiltonian is written as ${ }^{13,14}$

$$
H_{\text {Total }}=H_{\text {mol }}(D)+H_{\text {mol }}(A)+H_{\text {rad }}+H_{\text {int }}(D)+H_{\text {int }}(A)
$$

where $H_{m o l}(\xi), \xi=\mathrm{D}$, A are molecular Hamiltonians for the donor and acceptor composed of a sum of kinetic and intra-molecular potential energies for the charged particles that are grouped into distinct species $\xi$. In QED theory, the electromagnetic field is treated at the same level as the material particles, so an explicit Hamiltonian term is included from the very beginning. One form in which
$H_{\text {rad }}$ may be written is in terms of microscopic transverse electric displacement and magnetic fields, $\vec{d}^{\perp}(\vec{r})$ and $\vec{b}(\vec{r})$, respectively, as

$$
H_{\text {rad }}=\frac{1}{2} \int\left[\varepsilon_{0}^{-1} \vec{d}^{\perp 2}(\vec{r})+\varepsilon_{0} c^{2} \vec{b}^{2}(\vec{r})\right] d^{3} \vec{r},
$$

and represents the total energy of the electromagnetic field, which is viewed as a sum of independent simple harmonic oscillators.

Fourier series mode expansions are employed for the Maxwell field operators, whose free-space functional forms are

$$
\begin{aligned}
\vec{d}^{\perp}(\vec{r})= & i \sum_{\vec{k}, \lambda}\left(\frac{\hbar c k \varepsilon_{0}}{2 V}\right)^{1 / 2}\left[\vec{e}^{(\lambda)}(\vec{k}) a^{(\lambda)}(\vec{k}) e^{i \vec{k} \cdot \vec{r}}\right. \\
& \left.-\overline{\vec{e}}^{(\lambda)}(\vec{k}) a^{\dagger(\lambda)}(\vec{k}) e^{-i \vec{k} \cdot \vec{r}}\right]
\end{aligned}
$$

and

$$
\begin{aligned}
\vec{b}(\vec{r})= & i \sum_{\vec{k}, \lambda}\left(\frac{\hbar k}{2 \varepsilon_{0} c V}\right)^{1 / 2}\left[\vec{b}^{(\lambda)}(\vec{k}) a^{(\lambda)}(\vec{k}) e^{i \vec{k} \cdot \vec{r}}\right. \\
& \left.-\overline{\vec{b}}^{(\lambda)}(\vec{k}) a^{\dagger(\lambda)}(\vec{k}) e^{-i \vec{k} \cdot \vec{r}}\right]
\end{aligned}
$$

where the sum is executed over all modes of the radiation field specified by the wave vector $\vec{k}$ and the index of polarization $\lambda$, whose circular frequency is $\omega=c k$, for light in a box of volume $V$. The complex unit electric and magnetic polarization vectors are given by $\vec{e}^{(\lambda)}(\vec{k})$ and $\vec{b}^{(\lambda)}(\vec{k})=\hat{k} \times \vec{e}^{(\lambda)}(\vec{k})$ for $\vec{k}, \lambda$-mode radiation, with an overbar signifying the complex conjugate. The characteristic of the second quantized approach is the photon lowering and raising operators $a^{(\lambda)}(\vec{k})$ and $a^{\dagger(\lambda)}(\vec{k})$, which, respectively, decrease and increase the number of light quanta in the free radiation field by unity. A state of the field in which the number of quanta is equal to zero for each and every mode corresponds to the vacuum electromagnetic field. These individual bosonic shift operators obey the equal time commutator,

$$
\left[a^{(\lambda)}(\vec{k}), a^{\dagger\left(\lambda^{\prime}\right)}\left(\vec{k}^{\prime}\right)\right]=\frac{1}{8 \pi^{3} V} \delta_{\lambda \lambda^{\prime}} \delta\left(\vec{k}-\vec{k}^{\prime}\right),
$$

with all other commutator brackets involving these operators vanishing.

On taking the leading term in the multipolar expansion ${ }^{16}$ of the charge and current density distributions of the atoms or molecules results in the coupling between matter and radiation being given by the electric dipole approximated form of the interaction Hamiltonian,

$$
H_{\text {int }}(\xi)=-\varepsilon_{0}^{-1} \vec{\mu}(\xi) \cdot \vec{d}^{\perp}\left(\vec{R}_{\xi}\right),
$$

where $\vec{\mu}(\xi)$ is the electric dipole moment operator of species $\xi$.

For the situation in which $\mathrm{D}$ and $\mathrm{A}$ are embedded in a dielectric medium composed of individual microscopic particles other than the emitter and absorber, quantization of the bath Hamiltonian results in the free-space electric displacement field operator [Eq. (5)] being modified to 


$$
\begin{aligned}
\vec{d}^{\perp(m e d)}(\vec{r})= & i \sum_{\vec{p}, \eta}\left(\frac{\hbar \omega_{p}^{(v)} v_{g}^{(v)} \varepsilon_{0}}{2 c n^{(v)} V}\right)^{1 / 2}\left(\frac{\left(n^{(v)}\right)^{2}+2}{3}\right) \\
& \times\left[\vec{e}^{(\eta)}(\vec{p}) P_{v}^{(\eta)}(\vec{p}) e^{i \vec{p} \cdot \vec{r}}-\overline{\vec{e}}^{(\eta)}(\vec{p}) P_{v}^{\dagger(\eta)}(\vec{p}) e^{-i \vec{p} \cdot \vec{r}}\right]
\end{aligned}
$$

where the mode of the polariton is $\vec{p}, \eta$. In expression (9), $v$ is an index that specifies the polariton dispersion branch, $v_{g}^{(v)}$ is the group velocity of radiation propagating in the medium whose refractive index is $n^{(v)}$, and $P_{v}^{(\eta)}(\vec{p})$ and $P_{v}^{\dagger(\eta)}(\vec{p})$ are annihilation and creation operators, respectively, for a polariton of mode $\vec{p}, \eta$.

As in the free-space molecular QED calculation of the transfer matrix element, ${ }^{29}$ two time-ordered diagrams contribute to the probability amplitude, each depicting the directed exchange of a single virtual polariton between the donor and the acceptor. Using the second-order formula of time-dependent perturbation theory for the probability amplitude, the matrix element is found to be

$$
M_{f i}^{D A(m e d)}=\mu_{i}^{0 m}(D) V_{i j}^{(m e d)}\left(k_{m 0}, \vec{\rho}\right) \mu_{j}^{m 0}(A),
$$

where $\mu_{i}^{0 m}(\xi)$ is the transition electric dipole moment between states $\left|0^{\xi}\right\rangle$ and $\left|m^{\xi}\right\rangle$ of particle $\xi$. Note that the Einstein summation convention has been adopted in Eq. (10) for indices of Cartesian tensor components that repeat. In addition, appearing in Eq. (10) is the retarded electric dipole-electric dipole coupling tensor in a medium,

$$
V_{i j}^{(\text {med })}(p, \vec{R})=n^{-2}\left(\frac{n^{2}+2}{3}\right)^{2} V_{i j}^{(v a c)}(n p, \vec{R}),
$$

which is expressed in terms of the tensor coupling two electric dipoles separated by a vector displacement, $\vec{R}$, in free space, $V_{i j}^{\text {vac }}(p, \vec{R})$, whose functional form is ${ }^{13,14}$

$$
\begin{aligned}
V_{i j}^{(v a c)}(p, \vec{R}) & =-\frac{1}{4 \pi \varepsilon_{0}}\left(-\nabla^{2} \delta_{i j}+\nabla_{i} \nabla_{j}\right) \frac{e^{i p R}}{R} \\
& =\frac{e^{i p R}}{4 \pi \varepsilon_{0} R^{3}}\left[\left(\delta_{i j}-3 \hat{R}_{i} \hat{R}_{j}\right)(1-i p R)-\left(\delta_{i j}-\hat{R}_{i} \hat{R}_{j}\right) p^{2} R^{2}\right] .
\end{aligned}
$$

It is worth noting that due to the effect of an environment, the wave vector argument in the coupling tensor is scaled by $n=n^{\prime}+i n^{\prime \prime}$, the complex refractive index of the surrounding medium. This last quantity also appears in two explicit pre-factors of Eq. (11). The first is the screening factor and the second is the local field effect.

In the weak-coupling regime, the rate of resonant excitation energy transfer is given by the Fermi golden rule formula,

$$
\Gamma=\frac{2 \pi}{\hbar}\left|M_{f i}\right|^{2} \eta
$$

where $\eta$ is defined after Eq. (1). For a freely tumbling D-A pair, the rate in a medium is given by ${ }^{8,10,12}$

$$
\begin{aligned}
\left\langle\Gamma^{D A(m e d)}\right\rangle= & \frac{4 \pi \eta}{9 \hbar}\left|\frac{n^{2}+2}{3 n}\right|^{4}\left(4 \pi \varepsilon_{0} \rho^{3}\right)^{-2}\left|\vec{\mu}^{0 m}(D)\right|^{2}\left|\vec{\mu}^{m 0}(A)\right|^{2} e^{-2 n^{\prime \prime} k \rho} \\
& \times\left[3+6 n^{\prime \prime} k \rho+\left(n^{\prime 2}+5 n^{\prime \prime 2}\right) k^{2} \rho^{2}\right. \\
& \left.+2\left(n^{\prime 2}+n^{\prime \prime 2}\right) n^{\prime \prime} k^{3} \rho^{3}+|n|^{4} k^{4} \rho^{4}\right]
\end{aligned}
$$

where $k=k_{m 0}$ is the wavenumber of the downward transition in the donor particle. Equation (14) applies for all separations outside the orbital overlap region. Environmental effects such as screening and local field corrections are properly accounted for, as are dissipative effects in the medium via the exponential factor, whose argument displays a negative dependence on $n^{\prime \prime}$. On setting $n^{\prime}=1$ and $n^{\prime \prime}=0$, the result [Eq. (14)] expectedly reduces to its free-space form, Eq. (1).

The form and features of the rate expression [Eq. (14)] are a direct consequence of RET being viewed as due to the exchange of a virtual polariton between the emitter and the absorber within microscopic QED theory. It is interesting to note that within the framework of radiationless or Förster theory in a medium, the origin of the introduction of the refractive index has taken on a couple of different forms. One involves $n$ describing the intervening space between the two chromophores, in a localized picture. The Coulomb interaction is modified by an effective dielectric constant, which serves to screen the interaction. The other is the solvent picture, in which $n$ appears in the medium corrected emission intensity of $D$ and the absorption cross section of $A$. In this case, the relevant transition electric dipole moments are those for the chromophores within the medium, and not their free-space values.

\section{THIRD-BODY MEDIATION OF RET IN A FLUID}

We now examine the role of a neutral, polarizable but otherwise passive third body, $\mathrm{T}$, in modifying the rate of resonance energy transfer between $\mathrm{D}$ and $\mathrm{A}$ in a medium with a complex refractive index $n$. We can think of these three molecules as forming a quantum subsystem within a randomly oriented fluid environment; the latter comprises the "bath," which is fully described by its refractive index. We take all three molecules to be chemically identical; however, this can be relaxed if desired for generality.

With the introduction of the third particle, transfer of energy between $\mathrm{D}$ and A may occur indirectly via the mediator in addition to direct transfer. Overall, there are four possible energy transfer mechanisms comprising direct migration from $\mathrm{D}$ to $\mathrm{A}$ and three routes involving T. To maintain consistency with previous studies, ${ }^{1,41-43}$ we use the same convention; one route is designated DTA, in which $\mathrm{T}$ serves as a bridging species between $\mathrm{D}$ and $\mathrm{A}$, and the other two are denoted as TDA and DAT, in which the mediator couples only to D or only to A, respectively. For these last two possibilities to manifest, $\mathrm{T}$ must have a non-vanishing ground state permanent electric dipole moment, $\vec{\mu}^{00}(T)$; namely, the mediator is polar. As a consequence of this property, it is interesting to note that no net transfer of energy occurs between $\mathrm{T}$ and $\mathrm{D}$ in the TDA mechanism, or between A and T in the DAT route. 
Accounting for the four migration mechanisms when a third body is present means that the total probability amplitude for RET may be written as

$$
M_{f i}^{\text {Total }}=M^{D A}+M^{T D A}+M^{D A T}+M^{D T A} .
$$

Fourth-order diagrammatic time-dependent perturbation theory may be employed to calculate the matrix element for each of the contributions dependent upon T. Coupling between any pair of interacting particles is viewed as arising from the propagation of a single virtual polariton between the respective centers so that each mechanism involving the third body requires summation over 24 separate time-ordered sequences of (virtual) emission and absorption events. A representative graph for each of the three mechanisms is shown in Figs. 1(b)-1(d) of Ref. 1, from which the labeling of configurations either as TDA, DAT, or DTA becomes clear.

To account for the fact that the mediator $\mathrm{T}$ remains in its ground electronic state before and after the transfer process and to accurately represent the new total system, the initial and final states in Eq. (2) are modified by the inclusion of $\left|0^{T}\right\rangle$ in their specification. During the transfer process, the mediator may undergo virtual electronic transitions to an arbitrary state $\left|r^{T}\right\rangle$. As for pair transfer in a medium, the total system is assumed to be closed so that no energy is lost to the surroundings.

Because transfer is resonant and the chromophores are chemically equivalent, A's final state is also $\left|m^{A}\right\rangle$. It is worth noting that when $\mathrm{D}$ and $\mathrm{A}$ are chemically different, the requirement for transfer of energy to occur resonantly is that the emitter and absorber have overlapping energy spectra. However, in the present case, matrix elements taken over the same electronic states of the three species ensure that equal magnitudes of the electric dipole moment and polarizability operators ensue.

Formulae for the individual contributions to the total matrix element [Eq. (15)] that depend on $\mathrm{T}$ are computed using standard techniques of molecular QED theory, ${ }^{13,14}$ yielding

$$
\begin{aligned}
& M^{T D A}=\mu_{i}^{00}(T) V_{i j}^{m e d}(0, \vec{R}) \alpha_{j k}^{0 m}(D ;-k, 0) V_{k l}^{m e d}(k, \vec{\rho}) \mu_{l}^{m 0}(A), \\
& M^{D A T}=\mu_{i}^{0 m}(D) V_{i j}^{m e d}(k, \vec{\rho}) \alpha_{j k}^{m 0}(A ; 0, k) V_{k l}^{m e d}\left(0, \vec{R}^{\prime}\right) \mu_{l}^{00}(T),
\end{aligned}
$$

and

$$
M^{D T A}=\mu_{i}^{0 m}(D) V_{i j}^{m e d}(k, \vec{R}) \alpha_{j k}^{00}(T ;-k, k) V_{k l}^{m e d}\left(k, \vec{R}^{\prime}\right) \mu_{l}^{m 0}(A) .
$$

Environmental effects are included through the coupling tensor appropriate to a dielectric medium, Eq. (11). The separation distances between coupled particles are defined by the vectors $\vec{R}=\vec{R}_{D}-\vec{R}_{T}$ and $\vec{R}^{\prime}=\vec{R}_{A}-\vec{R}_{T}$ so that $\vec{\rho}=\vec{R}^{\prime}-\vec{R}$ is consistent with its definition given earlier. The appearance of a polarizability tensor in the expression for each amplitude is due to the scattering of a virtual polariton by the species which shuttles energy: a transition polarizability is associated with $\mathrm{D}$ and $\mathrm{A}$, respectively, in the case of the TDA and DAT mechanisms, and the ground state counterpart for $\mathrm{T}$ in the case of the DTA route. For any two-photon allowed molecular process taking place between states $|i\rangle$ and $|f\rangle$, the general functional form of the dynamic transition polarizability of particle $\xi$ is written as

$$
\alpha_{i j}^{f i}\left(\xi ;-p, p^{\prime}\right)=\sum_{r}\left[\frac{\mu_{i}^{f r}(\xi) \mu_{j}^{r i}(\xi)}{E_{r i}+\hbar c p+i \hbar c \gamma^{(r)}}+\frac{\mu_{j}^{f r}(\xi) \mu_{i}^{r i}(\xi)}{E_{r i}-\hbar c p^{\prime}+i \hbar c \gamma^{(r)}}\right],
$$

where the sum is executed over all intermediate states $\left|r^{\xi}\right\rangle$, and the damping of the molecule in this state is denoted by $\gamma^{(r)}$. It is important to point out that of the three polarizabilities featuring in the matrix elements Eqs. (16)-(18), only the ground state polarizability tensor of $\mathrm{T}$ is symmetric in its indices. The inverse length featuring in the arguments of the interaction tensor and polarizability correspond to the quantity of transferred energy, $E_{m 0}=E_{m}-E_{0}$ $=\hbar c k_{m 0}$, with $k_{m 0}=k$ chosen to simplify the notation. Performing the sum in Eq. (19) in a two-level approximation, the three relevant polarizabilities are found to be

$$
\begin{aligned}
\alpha_{i j}^{0 m}(D ;-k, 0)= & \frac{\mu_{i}^{00}(D) \mu_{j}^{0 m}(D)}{E_{m 0}+i \hbar c \gamma^{(0)}}+\frac{\mu_{j}^{00}(D) \mu_{i}^{0 m}(D)}{i \hbar c \gamma^{(0)}} \\
& +\frac{\mu_{i}^{0 m}(D) \mu_{j}^{m m}(D)}{i \hbar c \gamma^{(m)}}+\frac{\mu_{j}^{0 m}(D) \mu_{i}^{m m}(D)}{-E_{m 0}+i \hbar c \gamma^{(m)}}, \\
\alpha_{i j}^{m 0}(A ; 0, k)= & \frac{\mu_{i}^{m 0}(A) \mu_{j}^{00}(A)}{E_{m 0}+i \hbar c \gamma^{(0)}}+\frac{\mu_{j}^{m 0}(A) \mu_{i}^{00}(A)}{i \hbar c \gamma^{(0)}} \\
& +\frac{\mu_{i}^{m m}(A) \mu_{j}^{m 0}(A)}{i \hbar c \gamma^{(m)}}+\frac{\mu_{j}^{m m}(A) \mu_{i}^{m 0}(A)}{-E_{m 0}+i \hbar c \gamma^{(m)}}, \\
\alpha_{i j}^{00}(T ;-k, k)= & \frac{\mu_{i}^{00}(T) \mu_{j}^{00}(T)}{E_{m 0}+i \hbar c \gamma^{(0)}}+\frac{\mu_{j}^{00}(T) \mu_{i}^{00}(T)}{-E_{m 0}+i \hbar c \gamma^{(0)}} \\
& +\frac{\mu_{i}^{0 m}(T) \mu_{j}^{m 0}(T)}{i \hbar c \gamma^{(m)}}+\frac{\mu_{j}^{0 m}(T) \mu_{i}^{m 0}(T)}{-2 E_{m 0}+i \hbar c \gamma^{(m)}} .
\end{aligned}
$$

Since the three particles taken together are situated in the medium, it is appropriate to employ the vacuum polarizability of the mediator. The dispersion behavior of the environment, characterized by its refractive index, may be described via the Clausius-Mossotti relation and the bulk polarizability of the medium.

For coupling of polar $\mathrm{T}$, through its ground state permanent electric dipole moment in the TDA and DAT mechanisms, no net energy is relayed. Hence, the wavenumber argument appearing in $V_{i j}^{\text {med }}$ and the polarizability is zero in Eqs. (16) and (17), corresponding to the static or frequency-independent versions of these two quantities. Introducing the shorthand notation $\eta_{i j}^{\vec{r}(t)} \equiv \delta_{i j}-t \hat{r}_{i} \hat{r}_{j}$ for the orientational factors featuring in the right-hand side of Eq. (12), the five relevant coupling tensors present in the matrix elements (16)-(18) are 


$$
\begin{aligned}
V_{i j}^{\text {med }}(0, \vec{R})= & \frac{1}{4 \pi \varepsilon_{0} R^{3}}\left(\frac{n^{2}+2}{3 n}\right)^{2} \eta_{i j}^{\vec{R}(3)} \\
V_{i j}^{\text {med }}(k, \vec{\rho})= & \frac{1}{4 \pi \varepsilon_{0} \rho^{3}}\left(\frac{n^{2}+2}{3 n}\right)^{2} e^{i n^{\prime} k \rho} e^{-n^{\prime \prime} k \rho} \\
& \times\left[\eta_{i j}^{\vec{\rho}(3)}+n^{\prime \prime} k \rho \eta_{i j}^{\vec{\rho}(3)}+\left(n^{\prime \prime 2}-n^{\prime 2}\right) k^{2} \rho^{2} \eta_{i j}^{\vec{\rho}(1)}\right. \\
& \left.-i n^{\prime} k \rho \eta_{i j}^{\vec{\rho}(3)}-2 i n^{\prime} n^{\prime \prime} k^{2} \rho^{2} \eta_{i j}^{\vec{\rho}(1)}\right] \\
V_{i j}^{m e d}\left(0, \vec{R}^{\prime}\right)= & \frac{1}{4 \pi \varepsilon_{0} R^{\prime 3}}\left(\frac{n^{2}+2}{3 n}\right)^{2} \eta_{i j}^{\vec{R}^{\prime}(3)}, \\
V_{i j}^{m e d}(k, \vec{R})= & \frac{1}{4 \pi \varepsilon_{0} R^{3}}\left(\frac{n^{2}+2}{3 n}\right)^{2} e^{i n^{\prime} k R} e^{-n^{\prime \prime} k R} \\
& \times\left[\eta_{i j}^{\vec{R}(3)}+n^{\prime \prime} k R \eta_{i j}^{\vec{R}_{i j}(3)}+\left(n^{\prime \prime 2}-n^{\prime 2}\right) k^{2} R^{2} \eta_{i j}^{\vec{R}(1)}\right. \\
& \left.-i \eta^{\prime} k R \eta_{i j}^{\vec{R}(3)}-2 i \eta^{\prime} n^{\prime \prime} k^{2} R^{2} \eta_{i j}^{\vec{R}(1)}\right], \\
& \left.-i n^{\prime} k R^{\prime} \eta_{i j}^{\vec{R}^{\prime}(3)}-2 i n^{\prime} n^{\prime \prime} k^{2} R^{\prime 2} \eta_{i j}^{\vec{R}^{\prime}(1)}\right] \\
V_{i j}^{\text {med }}\left(k, \vec{R}^{\prime}\right)= & \frac{1}{4 \pi \varepsilon_{0} R^{\prime 3}}\left(\frac{n^{2}+2}{3 n}\right)^{2} e^{i n^{\prime} k R^{\prime}} e^{-n^{\prime \prime} k R^{\prime}} \\
& \times\left[\eta_{i j}^{\vec{R}^{\prime}(3)}+n^{\prime \prime} k R^{\prime} \eta_{i j}^{\vec{R}^{\prime}(3)}+\left(n^{\prime \prime 2}-n^{\prime 2}\right) k^{2} R^{\prime 2} \eta_{i j}^{\vec{R}^{\prime}(1)}\right.
\end{aligned}
$$

Inserting the total matrix element [Eq. (15)] into the Fermi golden rule rate formula [Eq. (13)] results in ten contributory terms,

$$
\begin{aligned}
\Gamma^{T o t a l}= & \frac{2 \pi \eta}{\hbar}\left[\left|M^{D A}\right|^{2}+2 \operatorname{Re}\left(M^{D A} \bar{M}^{T D A}\right)+\left|M^{T D A}\right|^{2}\right. \\
& +2 \operatorname{Re}\left(M^{D A} \bar{M}^{D A T}\right)+2 \operatorname{Re}\left(M^{T D A} \bar{M}^{D A T}\right)+\left|M^{D A T}\right|^{2} \\
& +2 \operatorname{Re}\left(M^{D A} \bar{M}^{D T A}\right)+2 \operatorname{Re}\left(M^{T D A} \bar{M}^{D T A}\right) \\
& \left.+2 \operatorname{Re}\left(M^{D A T} \bar{M}^{D T A}\right)+\left|M^{D T A}\right|^{2}\right]
\end{aligned}
$$

with the first term, corresponding to pair transfer in a medium, given by Eq. (14) for isotropic donor and acceptor molecules. An analysis of the dependence of the total rate on various contributions of Eq. (26) as well as the effect of interference of phase amplitudes, decoherence, and damping for a three-body system in a fixed relative orientation was recently given in Ref. 1. Below, we consider the case of isotropic species that may be situated arbitrarily relative to one another.

Before doing so, it is worth mentioning that interference effects for a process such as phonon mediated electronic excitation transfer that may occur directly between initial and final states, or via a third state or site, have been considered. ${ }^{60}$ A process is said to be sequential if the direct and third-state mediated routes are considered independent of one another, and their respective probabilities are summed in a "classical" fashion. This corresponds to a rapid thermalization of the bath. If thermalization is slow, however, then the probability is given by the modulus square of the sum of the amplitudes for the two routes. This is categorized as a "super-exchange" mechanism with coherence giving rise to a nonclassical interference term. Incidentally, we also note that there are a number of studies showing that interference effects are critically important when considering through-bond electron transfer, which also occurs via the super-exchange mechanism. ${ }^{61,62}$ In the present study, calculation of the total matrix element, Eq. (15), is carried out quantum electrodynamically. Furthermore, three different coupling mechanisms arise from taking proper account of the electronic properties of the mediator. There are interferences not only between different Feynman pathways from the various amplitudes associated with distinct third-body mediated mechanisms but also between direct and indirect processes. All of these contributions arise automatically in the present treatment and are contained in the result, Eq. (26).

We first evaluate diagonal (pure amplitude) contributions to the third-body mediated rate, Eq. (26), namely, those arising solely from the TDA, DAT, and DTA routes. To obtain results which hold for freely tumbling $\mathrm{D}, \mathrm{A}$, and $\mathrm{T}$, an orientational average ${ }^{63}$ is performed over the molecular quantities present in the rate formula. For averaging of the dipoles, we use the well-known result

$$
\left\langle\mu_{i}^{p q}(\xi) \bar{\mu}_{j}^{q p}(\xi)\right\rangle=\frac{1}{3} \delta_{i j}\left|\vec{\mu}^{p q}(\xi)\right|^{2}
$$

while for the rotationally averaged polarizability, we employ the expression $^{63}$

$$
\begin{aligned}
\left\langle\alpha_{k l}^{r s}(\xi) \bar{\alpha}_{k^{\prime} l^{\prime}}^{r s}(\xi)\right\rangle= & \frac{1}{30}\left\{\delta_{k l} \delta_{k^{\prime} l^{\prime}}\left[4 \alpha_{\lambda \lambda}^{r s}(\xi) \bar{\alpha}_{\mu \mu}^{r s}(\xi)-\alpha_{\lambda \mu}^{r s}(\xi) \bar{\alpha}_{\lambda \mu}^{r s}(\xi)-\alpha_{\lambda \mu}^{r s}(\xi) \bar{\alpha}_{\mu \lambda}^{r s}(\xi)\right]\right. \\
& +\delta_{k l^{\prime}} \delta_{k^{\prime} l}\left[-\alpha_{\lambda \lambda}^{r s}(\xi) \bar{\alpha}_{\mu \mu}^{r s}(\xi)-\alpha_{\lambda \mu}^{r s}(\xi) \bar{\alpha}_{\lambda \mu}^{r s}(\xi)+4 \alpha_{\lambda \mu}^{r s}(\xi) \bar{\alpha}_{\mu \lambda}^{r s}(\xi)\right] \\
& \left.+\delta_{k k^{\prime}} \delta_{l l^{\prime}}\left[-\alpha_{\lambda \lambda}^{r s}(\xi) \bar{\alpha}_{\mu \mu}^{r s}(\xi)+4 \alpha_{\lambda \mu}^{r s}(\xi) \bar{\alpha}_{\lambda \mu}^{r s}(\xi)-\alpha_{\lambda \mu}^{r s}(\xi) \bar{\alpha}_{\mu \lambda}^{r s}(\xi)\right]\right\}
\end{aligned}
$$

where the Greek subscripts signify Cartesian tensor components in the body-fixed (laboratory) frame of reference. We also require the result for the orientational average of a third-rank Cartesian tensor, ${ }^{63} T_{i j k}$,

$$
\left\langle T_{i j k}\right\rangle=\frac{1}{6} \varepsilon_{i j k} \varepsilon_{\lambda \mu v} T_{\lambda \mu \nu}
$$

as occurs in the product of a permanent electric dipole and a transition polarizability of a particular molecule. These tensors are then contracted with the retarded coupling tensors. The ensuing isotropic rate for the TDA mechanism is then 


$$
\begin{aligned}
\left\langle\Gamma^{T D A}\right\rangle= & \frac{\pi \eta}{135 \hbar} \frac{1}{\left(4 \pi \varepsilon_{0}\right)^{4}} \frac{1}{(\rho R)^{6}}\left|\left(\frac{n^{2}+2}{3 n}\right)^{2}\right|^{4}\left|\vec{\mu}^{00}(T)\right|^{2}\left|\vec{\mu}^{m 0}(A)\right|^{2} e^{-2 n^{\prime \prime} k \rho} \\
& \times\left\{\left[3 \alpha_{\lambda \lambda}^{0 m}(D ;-k, 0) \bar{\alpha}_{\mu \mu}^{m 0}(D ;-k, 0)-2 \alpha_{\lambda \mu}^{0 m}(D ;-k, 0) \bar{\alpha}_{\lambda \mu}^{m 0}(D ;-k, 0)+3 \alpha_{\lambda \mu}^{0 m}(D ;-k, 0) \bar{\alpha}_{\mu \lambda}^{m 0}(D ;-k, 0)\right]\right. \\
& \times\left(\left[9\left[1+(\hat{\rho} \cdot \hat{R})^{2}\right]\left[1+i(\bar{n}-n) k \rho+|n|^{2} k^{2} \rho^{2}\right]-\left[5-3(\hat{\rho} \cdot \hat{R})^{2}\right]\left[\left(n^{2}+\bar{n}^{2}\right) k^{2} \rho^{2}-i\left(n \bar{n}^{2}-\bar{n} n^{2}\right) k^{3} \rho^{3}-|n|^{4} k^{4} \rho^{4}\right)\right]\right) \\
& +12\left[-\alpha_{\lambda \lambda}^{0 m}(D ;-k, 0) \bar{\alpha}_{\mu \mu}^{m 0}(D ;-k, 0)+4 \alpha_{\lambda \mu}^{0 m}(D ;-k, 0) \bar{\alpha}_{\lambda \mu}^{m 0}(D ;-k, 0)-\alpha_{\lambda \mu}^{0 m}(D ;-k, 0) \bar{\alpha}_{\mu \lambda}^{m 0}(D ;-k, 0)\right] \\
& \left.\times\left[3+3 i(\bar{n}-n) k \rho+\left(3|n|^{2}-n^{2}-\bar{n}^{2}\right) k^{2} \rho^{2}+i\left(n \bar{n}^{2}-\bar{n} n^{2}\right) k^{3} \rho^{3}+|n|^{4} k^{4} \rho^{4}\right]\right\} .
\end{aligned}
$$

The rate result for the DAT mechanism may be obtained from expression (30) on replacing $R$ by $R^{\prime}, \hat{R}$ by $\hat{R}^{\prime}, \vec{\mu}^{m 0}(A)$ by $\vec{\mu}^{m 0}(D)$, and $\alpha_{\rho \sigma}^{0 m}(D ;-k, 0)$ by $\alpha_{\rho \sigma}^{0 m}(A ; 0, k)$. A lengthier expression is obtained for the isotropic rate due to the DTA route. It is given by

$$
\begin{aligned}
& \left\langle\Gamma^{D T A}\right\rangle=\frac{\pi \eta}{135 \hbar} \frac{1}{\left(4 \pi \varepsilon_{0}\right)^{4}} \frac{1}{\left(R R^{\prime}\right)^{6}}\left|\left(\frac{n^{2}+2}{3 n}\right)^{2}\right|^{4}\left|\vec{\mu}^{0 m}(D)\right|^{2}\left|\vec{\mu}^{m 0}(A)\right|^{2} e^{-2 n^{\prime \prime} k\left(R+R^{\prime}\right)}\left\{\left[3 \alpha_{\lambda \lambda}^{00}(T ;-k, k) \bar{\alpha}_{\mu \mu}^{00}(T ;-k, k)+\alpha_{\lambda \mu}^{00}(T ;-k, k) \bar{\alpha}_{\lambda \mu}^{00}(T ;-k, k)\right]\right. \\
& \times\left([ 1 + ( \hat { R } \cdot \hat { R } ^ { \prime } ) ^ { 2 } ] \left[9+9 i(\bar{n}-n) k\left(R+R^{\prime}\right)+9|n|^{2} k^{2}\left(R^{2}+R^{\prime 2}\right)-9(\bar{n}-n)^{2} k^{2} R R^{\prime}+9 i(\bar{n}-n)|n|^{2} k^{3} R R^{\prime}\left(R+R^{\prime}\right)\right.\right. \\
& +9|n|^{4} k^{4} R^{2} R^{\prime 2}+\left(n^{2}+\bar{n}^{2}\right)^{2} k^{4} R^{2} R^{\prime 2}-i\left(n^{2}+\bar{n}^{2}\right)\left(n \bar{n}^{2}-\bar{n} n^{2}\right) k^{5} R^{2} R^{\prime 2}\left(R+R^{\prime}\right)-\left(n \bar{n}^{2}-\bar{n} n^{2}\right)^{2} k^{6} R^{3} R^{\prime 3} \\
& \left.-\left(n^{2}+\bar{n}^{2}\right)|n|^{4} k^{6} R^{2} R^{\prime 2}\left(R^{2}+R^{\prime 2}\right)+i\left(n \bar{n}^{2}-\bar{n} n^{2}\right)|n|^{4} k^{7} R^{3} R^{\prime 3}\left(R+R^{\prime}\right)+|n|^{8} k^{8} R^{4} R^{\prime 4}\right] \\
& +\left[5-3\left(\hat{R} \cdot \hat{R}^{\prime}\right)^{2}\right]\left[\left(n^{2}+\bar{n}^{2}\right) k^{2}\left(R^{2}+R^{\prime 2}\right)+i(\bar{n}-n)\left(n^{2}+\bar{n}^{2}\right) k^{3} R R^{\prime}\left(R+R^{\prime}\right)-i\left(n \bar{n}^{2}-\bar{n} n^{2}\right) k^{3}\left(R^{3}+R^{\prime 3}\right)\right. \\
& +(\bar{n}-n)\left(n \bar{n}^{2}-\bar{n} n^{2}\right) k^{4} R R^{\prime}\left(R^{2}+R^{\prime 2}\right)+|n|^{2}\left(n^{2}+\bar{n}^{2}\right) k^{4} R^{\prime 2}\left(R^{2}+R^{\prime 2}\right)-|n|^{4} k^{4}\left(R^{4}+R^{\prime 4}\right) \\
& \left.\left.-i(\bar{n}-n)|n|^{4} k^{5} R R^{\prime}\left(R^{3}+R^{\prime 3}\right)-i|n|^{2}\left(n \bar{n}^{2}-\bar{n} n^{2}\right) k^{5} R^{2} R^{\prime 2}\left(R+R^{\prime}\right)-|n|^{6} k^{6} R^{2} R^{\prime 2}\left(R^{2}+R^{\prime 2}\right)\right]\right) \\
& +4\left[-\alpha_{\lambda \lambda}^{00}(T ;-k, k) \bar{\alpha}_{\mu \mu}^{00}(T ;-k, k)+3 \alpha_{\lambda \mu}^{00}(T ;-k, k) \bar{\alpha}_{\lambda \mu}^{00}(T ;-k, k)\right]\left[9+9 i(\bar{n}-n) k\left(R+R^{\prime}\right)+3\left(3|n|^{2}-n^{2}-\bar{n}^{2}\right) k^{2}\left(R^{2}+R^{\prime 2}\right)\right. \\
& -9(\bar{n}-n)^{2} k^{2} R R^{\prime}+3 i\left(n \bar{n}^{2}-\bar{n} n^{2}\right) k^{3}\left(R^{3}+R^{\prime 3}\right)+3 i(\bar{n}-n)\left(3|n|^{2}-n^{2}-\bar{n}^{2}\right) k^{3} R R^{\prime}\left(R+R^{\prime}\right) \\
& +3|n|^{4} k^{4}\left(R^{4}+R^{\prime 4}\right)-3(\bar{n}-n)\left(n \bar{n}^{2}-\bar{n} n^{2}\right) k^{4} R R^{\prime}\left(R^{2}+R^{\prime 2}\right)+\left(3|n|^{2}-n^{2}-\bar{n}^{2}\right)^{2} k^{4} R^{2} R^{\prime 2}+3 i(\bar{n}-n)|n|^{4} k^{5} R R^{\prime}\left(R^{3}+R^{\prime 3}\right) \\
& +i\left(3|n|^{2}-n^{2}-\bar{n}^{2}\right)\left(n \bar{n}^{2}-\bar{n} n^{2}\right) k^{5} R^{2} R^{\prime 2}\left(R+R^{\prime}\right)+\left(3|n|^{2}-n^{2}-\bar{n}^{2}\right)|n|^{4} k^{6} R^{2} R^{\prime 2}\left(R^{2}+R^{\prime 2}\right)-\left(n \bar{n}^{2}-\bar{n} n^{2}\right)^{2} k^{6} R^{3} R^{\prime 3} \\
& \left.\left.+i\left(n \bar{n}^{2}-\bar{n} n^{2}\right)|n|^{4} k^{7} R^{3} R^{\prime 3}\left(R+R^{\prime}\right)+|n|^{8} k^{8} R^{4} R^{4}\right]\right\} \text {. }
\end{aligned}
$$

Near- and far-zone limiting forms of the bridge-mediated transfer rate are readily obtained from Eq. (31), displaying characteristic inverse sixth and inverse square dependences on respective relative displacements; the former is indicative of a Förster type of radiationless energy migration, while the latter corresponds to a radiative transfer mechanism. Similar interpretations apply to the asymptotes of the other two pure contributions to the three-body rate.

Using Eqs. (10) and (16)-(18), the various interference terms contributing to the total rate [Eq. (26)] may be calculated. For the sake of completeness, these are explicitly given below. Of the six terms, only the $2 \operatorname{Re} \bar{M}^{D A} M^{D T A}$ and $2 \operatorname{Re} \bar{M}^{T D A} M^{D A T}$ contributions survive orientational averaging; the other four terms vanish. It is worth noting that the $2 \mathrm{Re} \bar{M}^{T D A} M^{D A T}$ contribution vanishes when all three molecules are identical. On defining the direction cosines formed from products of the unit displacement vectors as $u=\hat{\rho} \cdot \hat{R}, v=\hat{\rho} \cdot \hat{R}^{\prime}$, and $w=\hat{R} \cdot \hat{R}^{\prime}$, the first of these non-vanishing interference terms is

$$
\begin{aligned}
\left\langle\Gamma^{D A-D T A}\right\rangle= & \frac{4 \pi \eta}{3^{3} \bar{\hbar}} \frac{1}{\left(4 \pi \varepsilon_{0}\right)^{3}} \frac{1}{\left(\rho R R^{\prime}\right)^{3}} \operatorname{Re}\left|\left(\frac{n^{2}+2}{3 n}\right)^{2}\right|^{2}\left(\frac{n^{2}+2}{3 n}\right)^{2}\left|\vec{\mu}^{0 m}(D)\right|^{2}\left|\vec{\mu}^{m 0}(A)\right|^{2} \alpha(T ;-k, k) e^{i n k\left(R+R^{\prime}\right)} e^{-i \bar{n} k \rho} \\
& \times\left\{\left[6-9\left(u^{2}+v^{2}+w^{2}\right)+27 u v w\right](1-i n k R)\left(1-i n k R^{\prime}\right)(1+i \bar{n} k \rho)\right. \\
& +\left[-4+3\left(u^{2}+v^{2}+3 w^{2}\right)-9 u v w\right](1-i n k R)\left(1-i n k R^{\prime}\right) \bar{n}^{2} k^{2} \rho^{2} \\
& +\left[-4+3\left(3 u^{2}+v^{2}+w^{2}\right)-9 u v w\right](1-i n k R) n^{2} k^{2} R^{\prime 2}(1+i \bar{n} k \rho) \\
& +\left[-4+3\left(u^{2}+3 v^{2}+w^{2}\right)-9 u v w\right] n^{2} k^{2} R^{2}\left(1-i n k R^{\prime}\right)(1+i \bar{n} k \rho) \\
& -\left[-2+3 u^{2}+v^{2}+3 w^{2}-3 u v w\right](1-i n k R) n^{2} k^{2} R^{\prime 2} \bar{n}^{2} k^{2} \rho^{2} \\
& -\left[-2+u^{2}+3 v^{2}+3 w^{2}-3 u v w\right] n^{2} k^{2} R^{2}\left(1-i n k R^{\prime}\right) \bar{n}^{2} k^{2} \rho^{2} \\
& -\left[-2+3 u^{2}+3 v^{2}+w^{2}-3 u v w\right] n^{2} k^{2} R^{2} n^{2} k^{2} R^{\prime 2}(1+i n k \rho) \\
& \left.+\left[u^{2}+v^{2}+w^{2}-u v w\right] n^{2} k^{2} R^{2} n^{2} k^{2} R^{\prime 2} \bar{n}^{2} k^{2} \rho^{2}\right\}
\end{aligned}
$$


Asymptotic limits readily follow from Eq. (32). In the near-zone, we obtain

$$
\left\langle\Gamma^{D A-D T A}\right\rangle_{N Z}=\frac{4 \pi \eta}{9 \hbar} \frac{1}{\left(4 \pi \varepsilon_{0}\right)^{3}} \frac{1}{\left(\rho R R^{\prime}\right)^{3}} \operatorname{Re}\left|\left(\frac{n^{2}+2}{3 n}\right)^{2}\right|^{2}\left(\frac{n^{2}+2}{3 n}\right)^{2}\left|\vec{\mu}^{0 m}(D)\right|^{2}\left|\vec{\mu}^{m 0}(A)\right|^{2} \alpha(T ;-k, k)\left[2-3\left(u^{2}+v^{2}+w^{2}\right)+9 u v w\right],
$$

which exhibits inverse cube dependence on each of the distances. For the far-zone limit, the last line within braces of expression (32) is kept to yield the asymptote,

$$
\left\langle\Gamma^{D A-D T A}\right\rangle_{F Z}=\frac{4 \pi \eta}{27 \hbar} \frac{1}{\left(4 \pi \varepsilon_{0}\right)^{3}} \frac{k^{6}}{\rho R R^{\prime}} \operatorname{Ren} n^{4} \bar{n}^{2}\left|\left(\frac{n^{2}+2}{3 n}\right)^{2}\right|^{2}\left(\frac{n^{2}+2}{3 n}\right)^{2}\left|\vec{\mu}^{0 m}(D)\right|^{2}\left|\vec{\mu}^{m 0}(A)\right|^{2} \alpha(T ;-k, k) e^{i n k\left(R+R^{\prime}\right)} e^{-i \bar{n} k \rho}\left[u^{2}+v^{2}+w^{2}-u v w\right],
$$

which displays inverse dependence on each displacement variable. On inserting $n=1$, results [(33) and (34)] reduce to previously obtained limiting forms. ${ }^{42}$

The other non-vanishing interference term arises from the TDA and DAT mechanisms and is obtained from matrix elements (16) and (17), and using Eq. (29), yielding

$$
\begin{aligned}
\left\langle\Gamma^{T D A-D A T}\right\rangle= & \frac{4 \pi \eta}{108 \hbar} \operatorname{Re}\left|\frac{n^{2}+2}{3 n}\right|^{8} \frac{1}{\left(4 \pi \varepsilon_{0}\right)^{4}} \frac{1}{R^{3} R^{\prime 3} \rho^{6}}\left|\vec{\mu}^{00}(T)\right|^{2} \varepsilon_{\lambda \mu v} \varepsilon_{\rho \sigma \tau} \mu_{\lambda}^{0 m}(D) \bar{\alpha}_{\mu v}^{m 0}(D ;-k, 0) \bar{\mu}_{\rho}^{0 m}(A) \alpha_{\sigma \tau}^{m 0}(A ; 0, k) \\
& \times\left\{18\left[-1+u^{2}+v^{2}+w^{2}-3 u v w\right]\left(1+i(\bar{n}-n) k \rho+|n|^{2} k^{2} \rho^{2}+|n|^{4} k^{4} \rho^{4}\right)+18 w^{2}\left(3\left[1+i(\bar{n}-n) k \rho+|n|^{2} k^{2} \rho^{2}\right]+|n|^{4} k^{4} \rho^{4}\right)\right. \\
& +2\left\{19-21\left[u^{2}+v^{2}+w^{2}\right]+36 u v w\right\}\left(n^{2} k^{2} \rho^{2}+i n^{2} \bar{n} k^{3} \rho^{3}\right)+2\left\{13-12\left[u^{2}+v^{2}+w^{2}\right]+36 u v w\right\} \\
& \left.\times\left(\bar{n}^{2} k^{2} \rho^{2}-i n \bar{n}^{2} k^{3} \rho^{3}\right)-30 w^{2}\left[\left(n^{2}+\bar{n}^{2}\right) k^{2} \rho^{2}+i n \bar{n}(n-\bar{n})\right]\right\} e^{i k(n-\bar{n}) \rho} .
\end{aligned}
$$

\section{QUANTUM COHERENCE IN A FLUID}

There are two material parameters that directly influence RET efficiency, independent of the positions and orientations of the three molecules: polarizability damping $\gamma^{(r)}$ and the imaginary part of refractive index $n^{\prime \prime}$ (i.e., opacity). The damping may be physically attributed to vibronic coupling, that is to say, while in some electronically excited state $|r\rangle$, a molecule is able to exchange energy through its vibrational degrees of freedom, with energy carried away from the system as polaritons. Within the QED framework, which is formally closed, this appears as non-Hermitian terms in the Hamiltonian. The imaginary part of the refractive index $n^{\prime \prime}$ also gives rise to non-Hermiticity and can be understood physically as virtual polaritons becoming absorbed by the solvent bath.

It is interesting to consider how amplitude and phase coherences are manifested in a fluid such as a liquid, compared to oriented systems, such as static crystal lattices or quasi-static architectures as in natural light-harvesting systems. Noting the index nonsymmetry properties of the transition polarizabilities of the donor and acceptor, while the ground state polarizability of the mediator, $\mathrm{T}$, is index symmetric [see Eq. (20) and the Appendix], the TDADTA and DAT-DTA cross terms vanish on orientational averaging. Moreover, the DA-TDA and DA-DAT cross terms do not survive rotational averaging because the I-1 average of the ground state permanent electric dipole moment of $\mathrm{T}$ vanishes. The DADTA interference term on the other hand survives isotropic averaging (rank 2 average for transition dipole-squared of D and A and rank 4 average of polarizability of T) and is given by Eq. (32). Furthermore, the orientational average of the TDA-DAT cross term is non-vanishing: matrix elements are given by Eqs. (16) and (17); a rank 2 average features for permanent dipoles squared of $\mathrm{T}$, along with a product of two rank 3 averages for the transition dipole and the transition polarizability for each of the donor and the acceptor species, which does not vanish because the transition polarizabilities are not index symmetric (see the Appendix). This term is given by Eq. (35). In summary, of the ten rate terms, the four pure terms and two of the cross terms (DA-DTA and TDA-DAT) survive orientational averaging, and four cross terms do not.

\section{COMPUTATIONAL SIMULATIONS}

In this section, we compute the isotropic rates for the TDA and DAT mechanisms obtained from Eq. (30) and the isotropic rates for the DTA, DA-DTA, and TDA-DAT mechanisms from Eqs. (31), (32), and (35), respectively, for a series of model systems in order to understand the role of the different pure-amplitude and interference cross terms appearing in Eq. (26), which have both phase and amplitude components. All codes were developed in Python 3.5 employing the usual NumPy and SciPy libraries. In the simulations, we assume a transition frequency of $6.32 \times 10^{-19} \mathrm{~J}$ and that all three species are identical with permanent and transition dipole moments of magnitude of $3.33 \times 10^{-29} \mathrm{Cm}$, but with the permanent dipoles parallel to the $\mathrm{x}$-axis with the unit vector (1.0, $0.0,0.0)$ and the transition dipoles having a unit vector of $(0.33$, $0.33,0.33)$. A refractive index of $n=1.1+0.1 i$ was used for the medium, and all damping factors correspond to a magnitude of 0.2 of the mediating wave vector appearing in the polarizability equations.

Figure 1 shows the four different configurations for mediated RET. The dashed lines represent the coordinates varied as indicated by the abscissa axes in Figs. 2-4.

Figures 2-4 give quantitative insight into the roles that different pure-amplitude and interference terms play in mediated RET within an isotropic fluid. Figure 2 displays the total rate and the individual contributions from Eq. (26) for RET in the case of a linear DTA system, as shown in Fig. 1(a). The subplot in Fig. 2(a) displays the 


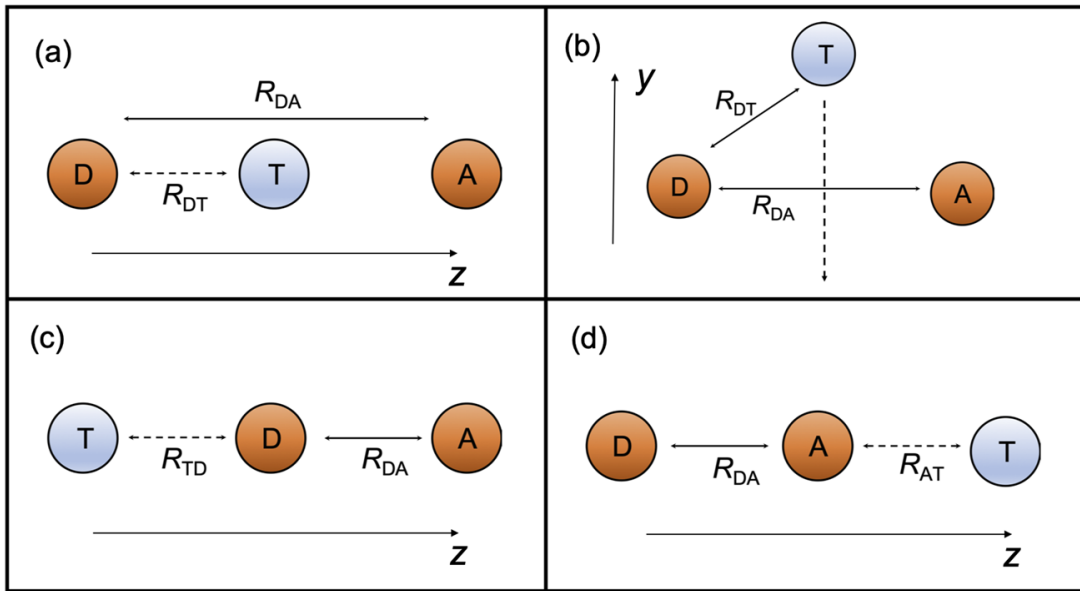

FIG. 1. Geometries of the DTA configurations that we employ for our model system. (a) The mediating chromophore $T$ is between $D$ and $A$, with all three species collinear, (b) DTA takes on a triangular geometry with $T$ being moved down the vertical y-axis, and [(c) and (d)] with the mediator, T outside DA. The dashed lines represent the variable coordinate. (a)

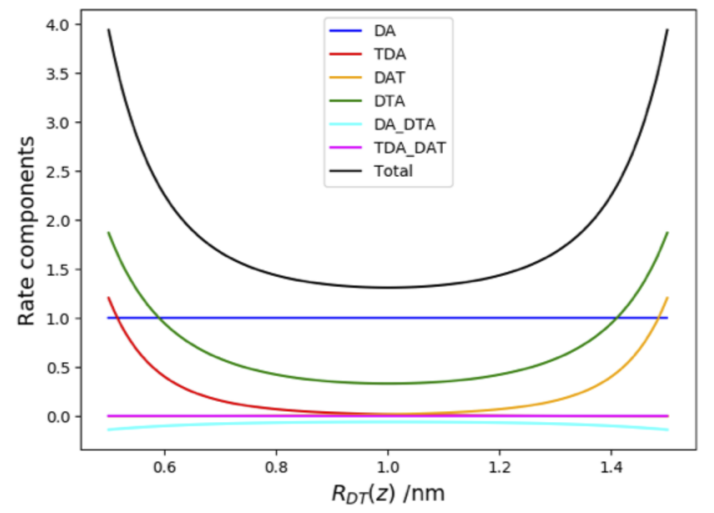

(b)

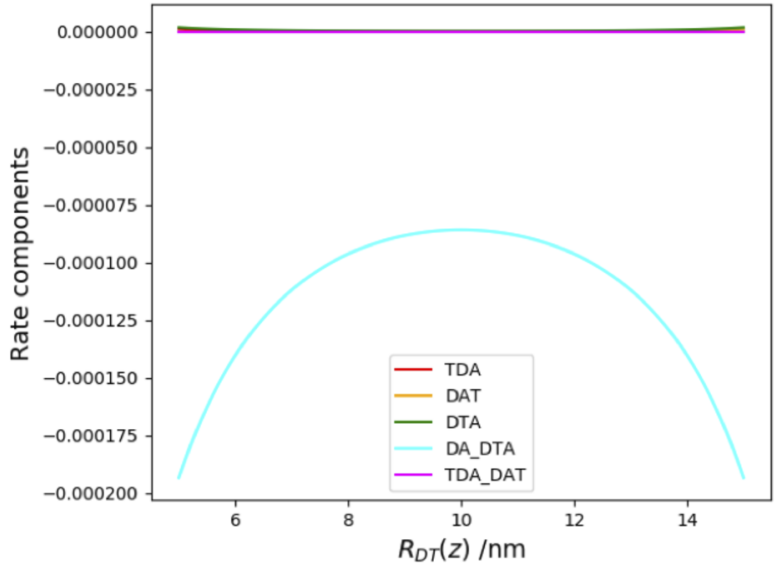

FIG. 2. Total relative rate and individual contributions to the relative rate from Eq. (26) for the collinear model system in Fig. 1 (a), for (a) $R_{\mathrm{DA}}=2 \mathrm{~nm}$ (near-zone) and (b) $R_{\mathrm{DA}}=20 \mathrm{~nm}$ (far-zone), normalized to the direct (DA) rate of RET. (a)

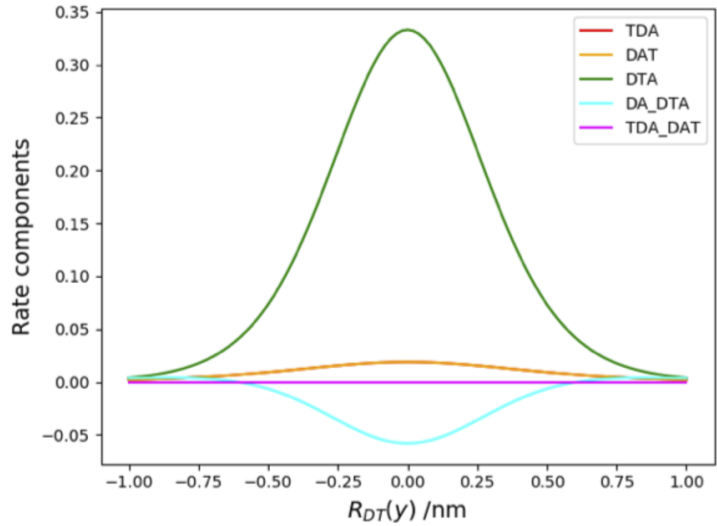

(b)

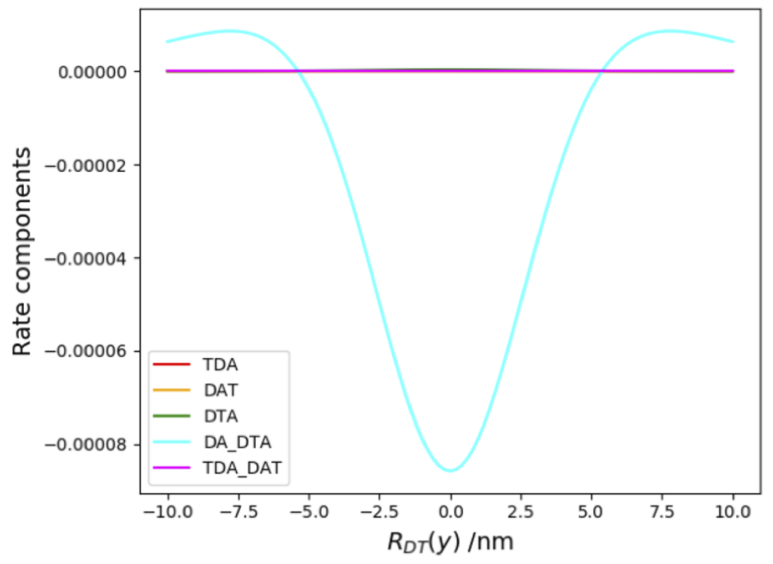

FIG. 3. Contributions to the relative rate from Eq. (26) for the model system with a triangular geometry in Fig. 1 (b) for (a) $R_{\mathrm{DA}}=2 \mathrm{~nm}$ (near-zone) and (b) $R_{\mathrm{DA}}=20 \mathrm{~nm}$ (far-zone), normalized to the direct (DA) rate of RET. 
(a)

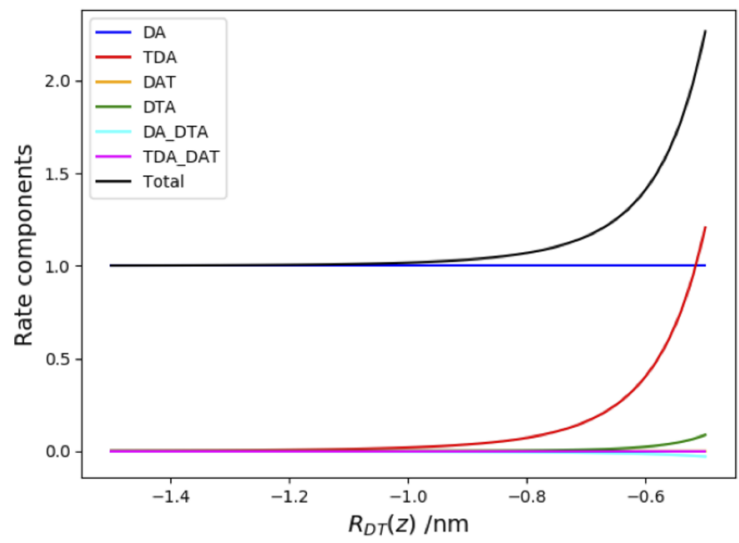

(b)

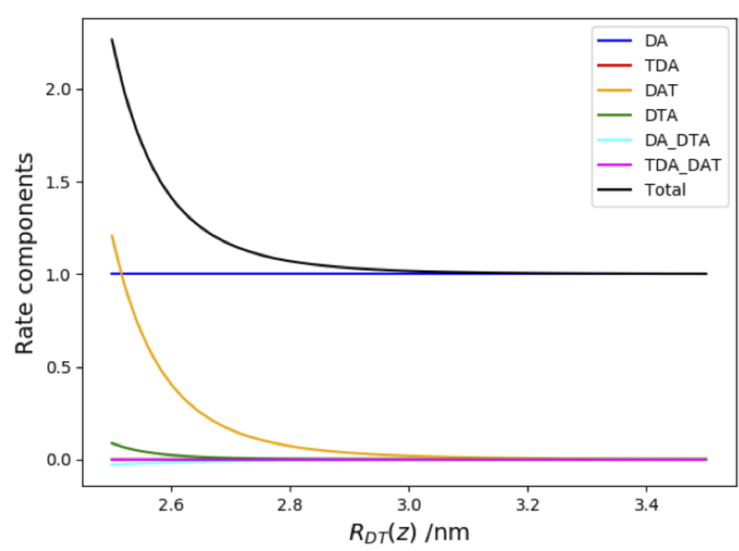

FIG. 4. Total relative rate and individual contributions to the relative rate from Eq. (26). (a) Model system in Fig. 1(c). (b) Model system in Fig. 1(d). $R_{\mathrm{DA}}=2 \mathrm{~nm}$ in both cases, with terms normalized to the direct (DA) rate of RET.

dependencies of the rate contributions in the near-zone, where D and $\mathrm{A}$ are $2 \mathrm{~nm}$ apart, and the relative distance $\mathrm{D}-\mathrm{T}$ is varied. As can be seen, the dominant contribution to the rate is the pure-DTA term, where $T$ directly mediates the transfer from $D$ to $A$. In the farzone, where D and A are $20 \mathrm{~nm}$ apart, Fig. 2(b), the interference cross term DA-DTA is the only one that contributes (albeit obviously at a much-reduced magnitude because of the increased distance between the chromophores). This is also reflected in the triangular model system, Fig. 1(b), where both the DTA and the DA-DTA interference terms make significant contributions to the isotropic rate equations, as can be seen in Fig. 3. The third model system we consider is a collinear one, but with the third chromophore outside the DA pair. In this case, we can see that the pure-TDA and pure-DAT terms dominate in a symmetric way, depending on whether the mediator is adjacent to the donor or the acceptor. This aspect is nicely illustrated in Fig. 4.

\section{DISCUSSION AND CONCLUSIONS}

In this work, we considered RET of a donor, acceptor, and third-body mediated system within an isotropic and disordered fluid. We used analytical rotational averaging techniques to consider how individual contributions of pure-amplitude terms and interference cross terms of the matrix element affected the rate of RET for this particular scenario. On orientational averaging, four of the cross terms vanish, including TDA-DTA, DAT-DTA, DATDA, and DA-DAT contributions. This highlights the fact that disordered environments give rise to strong quantum decoherence. Nevertheless, two cross terms survive, namely, DA-DTA, which involves both direct and mediated RET, where $\mathrm{T}$ bridges the donor and the acceptor, and one isotropic third-body mediated cross term, the TDA-DAT component, where $\mathrm{T}$ is on the outer side of the coupled DA pair.

Through computer simulations, we are able to gain quantitative insight into the relative contributions of the pure-amplitude and the phase cross terms to the total rate, for a series of distances. This enables a deeper understanding to be gained regarding radiationless and radiative energy transfer mechanisms, which are associated with near- and far-zone separation distance limits in the QED theory of RET. Within the quantum field description of RET adopted in the present study, both the chromophores and mediating fields (i.e., photons/polaritons) are treated quantum mechanically. Importantly, the nature of the mediating photon changes as the distance between the chromophores becomes larger. It is well known that, within a Coulomb gauge description of QED, ${ }^{13,14,16}$ at short distances (i.e., in the near-zone), the mediating photon has virtual character and, hence, has ill-defined physical properties, such as wavelength. Furthermore, in this distance regime, the photonic fields have significant longitudinal as well as transverse components. As the distance between chromophores increases, the photon becomes more real, and the longitudinal contributions to the field disappear leaving its transverse part to dominate. These aspects, coupled to the fact that phase offsets will begin to appear after several nanometers, explain why phase cross terms dominate at large distances, while pure-amplitude terms do so in the near-zone.

This work highlights how quantum coherence in RET arises via interference both between direct and indirect pathways, and also within the mediated mechanism. It is only through the existence of different pathways that this microscopic description of coherence can be understood in the same context as prototypical examples of quantum coherence, such as the quantum variant of the Young double-slit experiment. In this experiment, the existence of different trajectories through alternative slits allows for the emergence of interference between Feynman pathways. ${ }^{64}$ By understanding the origin of coherence in RET systems in this way, we can start to investigate mechanisms of decoherence for both amplitude and phase damping, going beyond standard phenomenological models.

\section{ACKNOWLEDGMENTS}

Professor D. L. Andrews and Dr. K. A. Forbes are thanked for helpful comments on the manuscript. The research presented in this paper was carried out on the High Performance Computing Cluster supported by the Research and Specialist Computing Support service at the University of East Anglia.

\section{APPENDIX: SYMMETRY PROPERTIES OF THE POLARIZABILITY TENSOR}

As detailed in Sec. VI, the TDA-DAT rate contribution does not in general vanish; however, it is very close to zero in the 
above simulations, due to the fact that the chromophores are chemically identical and because of the symmetry imposed on them geometrically.

In this appendix, we examine the index symmetry properties of the polarizability tensor. The general form of the dynamic electric dipole transition polarizability, including damping, is

$$
\alpha_{i j}^{t s}\left(\xi ; \pm p, \pm p^{\prime}\right)=\sum_{r}\left\{\frac{\mu_{i}^{t r}(\xi) \mu_{j}^{r s}(\xi)}{E_{r s} \mp h c p+i \hbar c \gamma^{r}}+\frac{\mu_{j}^{t r}(\xi) \mu_{i}^{r s}(\xi)}{E_{r s} \mp h c p^{\prime}+i \hbar c \gamma^{r}}\right\} .
$$

The transition polarizability appearing in the matrix element for the TDA mechanism is

$$
\alpha_{j k}^{0 m}(D ;-k, 0)=\sum_{r}\left\{\frac{\mu_{j}^{0 r}(D) \mu_{k}^{r m}(D)}{E_{r m}+h c k+i \hbar c \gamma^{r}}+\frac{\mu_{k}^{0 r}(D) \mu_{j}^{r m}(D)}{E_{r m}+i \hbar c \gamma^{r}}\right\} .
$$

For an arrangement of dipole moments such as that used in Sec. V, this polarizability is not symmetric in $j$ and $k$; i.e., interchanging $j$ and $k$ does not produce the same tensor.

The transition polarizability appearing in the matrix element for the DAT mechanism is

$$
\alpha_{j k}^{m 0}(A ; 0, k)=\sum_{r}\left\{\frac{\mu_{j}^{m r}(A) \mu_{k}^{r 0}(A)}{E_{r 0}+i \hbar c \gamma^{r}}+\frac{\mu_{k}^{m r}(A) \mu_{j}^{r 0}(A)}{E_{r 0}-\hbar c k+i \hbar c \gamma^{r}}\right\} .
$$

This is also not symmetric in $j$ and $k$.

The polarizability featuring in the matrix element for the DTA mechanism is a ground state polarizability of the form

$$
\alpha_{j k}^{00}(T ;-k,+k)=\sum_{r}\left\{\frac{\mu_{j}^{0 r}(T) \mu_{k}^{r 0}(T)}{E_{r 0}+h c k+i \hbar c \gamma^{r}}+\frac{\mu_{k}^{0 r}(T) \mu_{j}^{r 0}(T)}{E_{r 0}-h c k+i \hbar c \gamma^{r}}\right\},
$$

which is symmetric in $j, k$.

Let us now examine the case of zero damping and insert $\gamma^{r}=0$ in Eq. (A4). Then,

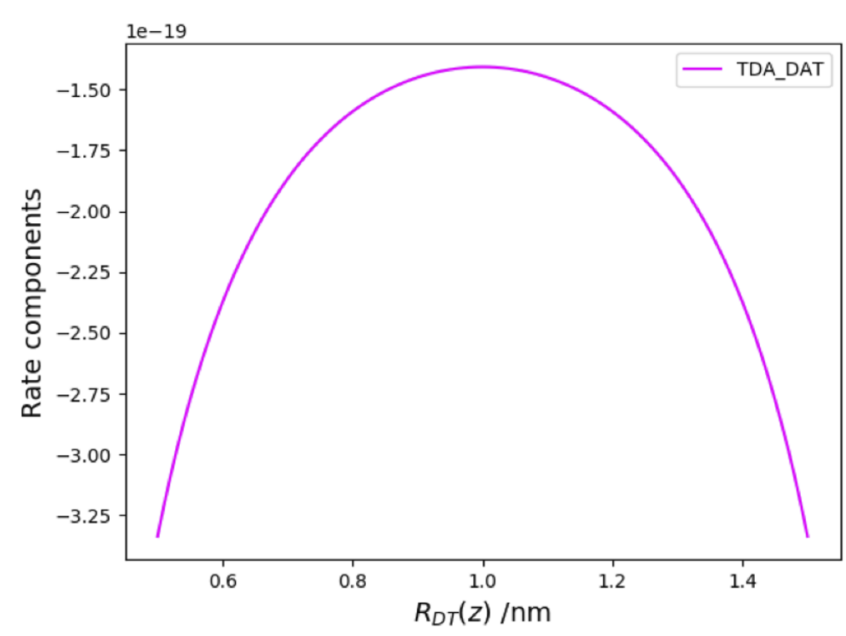

FIG. 5. A zoomed-in version of the TDA-DAT contribution to the rate from Fig. 2(a).

$$
\begin{aligned}
\alpha_{j k}^{00}(T ;-k,+k) & =\sum_{r}\left\{\frac{\mu_{j}^{0 r}(T) \mu_{k}^{r 0}(T)}{E_{r 0}+h c k}+\frac{\mu_{k}^{0 r}(T) \mu_{j}^{r 0}(T)}{E_{r 0}-h c k}\right\} \\
& =\sum_{r} \mu_{j}^{0 r}(T) \mu_{k}^{r 0}(T)\left\{\frac{1}{E_{r 0}+h c k}+\frac{1}{E_{r 0}-h c k}\right\} \\
& =2 \sum_{r} \frac{E_{r 0} \mu_{j}^{0 r}(T) \mu_{k}^{r 0}(T)}{E_{r 0}^{2}-(\hbar c k)^{2}},
\end{aligned}
$$

confirming the $j, k$-index symmetry, using $\vec{\mu}^{0 r}=\vec{\mu}^{r 0}$.

Hence, the isotropic rate for the TDA-DAT cross term, Eq. (35), is non-vanishing as it contains both polarizabilities Eqs. (A2) and (A3). However, if either one or both of these are symmetric in their indices, then the rate is zero because of the anti-symmetric epsilon tensors that appear. In Fig. 5, we show the small, but finite magnitude for TDA-DAT for the linear case of chromophores displayed in Fig. 1(a).

\section{REFERENCES}

${ }^{1}$ J. S. Ford, A. Salam, and G. A. Jones, "A quantum electrodynamics description of quantum coherence and damping in condensed phase energy transfer," J. Phys. Chem. Lett. 10, 5654-5661 (2019).

${ }^{2}$ H.-P. Breuer and F. Petruccione, The Theory of Open Quantum Systems (Oxford University Press, Oxford, 2007).

${ }^{3}$ R. S. Knox and H. van Amerongen, "Refractive index dependence of the Förster resonance excitation transfer rate," J. Phys. Chem. B 106, 5289-5293 (2002).

${ }^{4}$ G. D. Scholes, C. Curutchet, B. Mennucci, R. Cammi, and J. Tomasi, "How solvent controls electronic energy transfer and light harvesting," J. Phys. Chem. B 111, 6978-6982 (2007).

5 J. Tomasi, B. Mennucci, and R. Cammi, "Quantum mechanical solvation models," Chem. Rev. 105, 2999-3093 (2005).

${ }^{6}$ F. Lipparini and B. Mennucci, "Perspective: Polarizable continuum models for quantum-mechanical descriptions," J. Chem. Phys. 144, 160901 (2016).

${ }^{7}$ J. Knoester and S. Mukamel, "Intermolecular forces, spontaneous emission, and superradiance in a dielectric medium: Polariton-mediated interactions," Phys. Rev. A 40, 7065-7080 (1989).

${ }^{8}$ G. Juzeliunas and D. L. Andrews, "Quantum electrodynamics of resonant energy transfer in condensed matter," Phys. Rev. B 49, 8751-8763 (1994).

${ }^{9} \mathrm{M}$. Agranovitch and A. A. Maradudin, Electronic Excitation Energy Transfer in Condensed Matter (North-Holland, Amsterdam, 1982).

${ }^{10} \mathrm{G}$. Juzeliunas and D. L. Andrews, "Quantum electrodynamics of resonance energy transfer,” Adv. Chem. Phys. 112, 357-410 (2000).

${ }^{11}$ V. May and O. Kühn, Charge and Energy Transfer Dynamics in Molecular Systems (Wiley VCH, Weinheim, 2011).

${ }^{12}$ M. P. E. Lock, D. L. Andrews, and G. A. Jones, "On the nature of longrange electronic coupling in a medium: Distance and orientational dependence for chromophores in molecular aggregates," J. Chem. Phys. 140, 044103 (2014).

${ }^{13}$ D. P. Craig and T. Thirunamachandran, Molecular Quantum Electrodynamics (Dover, New York, 1998).

${ }^{14}$ A. Salam, Molecular Quantum Electrodynamics (John Wiley \& Sons, Inc., Hoboken, 2010).

${ }^{15}$ A. Salam, "Quantum electrodynamics effects in atoms and molecules," Wiley Interdiscip. Rev.: Comput. Mol. Sci. 5, 178-201 (2015).

${ }^{16}$ D. L. Andrews, G. A. Jones, A. Salam, and R. G. Woolley, "Perspective: Quantum Hamiltonians for optical interactions," J. Chem. Phys. 148, 040901 (2018).

${ }^{17}$ D. L. Andrews, D. S. Bradshaw, K. A. Forbes, and A. Salam, "Quantum electrodynamics in modern optics and photonics," J. Opt. Soc. Am. B 37, 1153-1172 (2020).

${ }^{18}$ R. R. McLone and E. A. Power, "On the interaction between two identical neutral dipole systems, one in the excited state and the other in the ground state," Mathematika 11, 91-94 (1964). 
${ }^{19}$ J. S. Avery, "Resonance energy transfer and spontaneous photon emission," Proc. Phys. Soc. 88, 1-8 (1966).

${ }^{20}$ E. A. Power and T. Thirunamachandran, "Quantum electrodynamics with nonrelativistic sources. III. Intermolecular interactions," Phys. Rev. A 28, 2671-2675 (1983).

${ }^{21}$ D. L. Andrews, "A unified theory of radiative and radiationless molecular energy transfer," Chem. Phys. 135, 195-210 (1989).

${ }^{22}$ A. K. Biswas, G. Compagno, G. M. Palma, R. Passante, and F. Persico, "Virtual photons and causality in the dynamics of a pair of two-level atoms," Phys. Rev. A 42, 4291-4301 (1990).

${ }^{23} \mathrm{E}$. A. Power and T. Thirunamachandran, "Analysis of the causal behaviour in energy transfer between atoms," Phys. Rev. A 56, 3395-3408 (1997).

${ }^{24}$ G. D. Scholes, "Long-range resonance energy transfer in molecular systems," Annu. Rev. Phys. Chem. 54, 57-87 (2003).

${ }^{25}$ G. J. Daniels, R. D. Jenkins, D. S. Bradshaw, and D. L. Andrews, "Resonance energy transfer: The unified theory revisited," J. Chem. Phys. 119, 2264-2274 (2003).

${ }^{26}$ A. Salam, "Resonant transfer of excitation between two molecules using Maxwell fields," J. Chem. Phys. 122, 044113 (2005).

${ }^{27}$ A. Salam, "Molecular quantum electrodynamics in the Heisenberg picture: A field theoretic viewpoint," Int. Rev. Phys. Chem. 27, 405-448 (2008).

${ }^{28} \mathrm{R}$. Grinter and G. A. Jones, "Resonance energy transfer: The unified theory via vector spherical harmonics," J. Chem. Phys. 145, 074107 (2016).

${ }^{29} \mathrm{~A}$. Salam, "The unified theory of resonance energy transfer according to molecular quantum electrodynamics," Atoms 6, 56 (2018).

${ }^{30}$ P. C. Nelson, "The role of quantum decoherence in FRET," Biophys. J. 115, 167-172 (2018).

${ }^{31}$ G. A. Jones and D. S. Bradshaw, "Resonance energy transfer: From fundamental theory to recent applications," Front. Phys. 7, 100 (2019).

${ }^{32}$ L.-Y. Hsu, W. Ding, and G. C. Schatz, "Plasmon-coupled resonance energy transfer," J. Phys. Chem. Lett. 8, 2357-2367 (2017).

${ }^{33}$ W. Ding, L.-Y. Hsu, and G. C. Schatz, "Plasmon-coupled resonance energy transfer: A real-time electrodynamics approach,” J. Chem. Phys. 146, 064109 (2017).

${ }^{34}$ K. N. Avanki, W. Ding, and G. C. Schatz, "Resonance energy transfer in arbitrary media: Beyond the point dipole approximation," J. Phys. Chem. C 122, 29445-29456 (2018).

${ }^{35}$ C.-P. Hsu, G. R. Fleming, M. Head-Gordon, and T. Head-Gordon, "Excitation energy transfer in condensed media," J. Chem. Phys. 114, 3065-3072 (2001).

${ }^{36}$ S. Caprasecca, C. Curutchet, and B. Mennucci, "Toward a unified modelling of environment and bridge-mediated contributions to electronic energy transfer: A fully polarisable QM/MM/PCM approach," J. Chem. Theory Comput. 8, 44624473 (2012).

${ }^{37}$ S. Caprasecca and B. Mennucci, "Excitation energy transfer in donor-bridgeacceptor systems: A combined quantum-mechanical/classical analysis of the role of the bridge and the solvent," J. Phys. Chem. A 118, 6484-6491 (2014).

${ }^{38}$ D. Weeraddana, M. Premaratne, and D. L. Andrews, "Direct and third-body mediated resonance energy transfer in dimensionally constrained nanostructures," Phys. Rev. B 92, 035128 (2015).

${ }^{39}$ D. Weeraddana, M. Premaratne, and D. L. Andrews, "Quantum electrodynamics of resonance energy transfer in nanowire systems," Phys. Rev. B 93, 075151 (2016).

${ }^{40}$ D. P. Craig and T. Thirunamachandran, "Third-body mediation of resonance coupling between identical molecules," Chem. Phys. 135, 37-48 (1989).

${ }^{41}$ G. J. Daniels and D. L. Andrews, "The electronic influence of a third-body on resonance energy transfer," J. Chem. Phys. 116, 6701-6712 (2002).

${ }^{42}$ A. Salam, "Mediation of resonance energy transfer by a third molecule," J. Chem. Phys. 136, 014509 (2012).
${ }^{43}$ D. L. Andrews and J. S. Ford, "Resonance energy transfer: Influence of neighbouring matter absorbing in the wavelength region of the acceptor," J. Chem. Phys. 139, 014107 (2013).

${ }^{44}$ R. D. Jenkins and D. L. Andrews, "Three-centre systems for energy pooling: Quantum electrodynamical theory," J. Phys. Chem. A 102, 10834-10842 (1998).

${ }^{45}$ D. L. Andrews and R. D. Jenkins, "A quantum electrodynamical theory of threecentre energy transfer for upconversion and downconversion in rare earth doped materials," J. Chem. Phys. 114, 1089-1100 (2001).

${ }^{46}$ M. D. LaCount, D. Weingarten, N. Hu, S. E. Shaheen, J. van de Lagemaat, G. Rumbles, D. M. Walba, and M. T. Lusk, "Energy pooling upconversion in organic molecular systems," J. Phys. Chem. A 119, 4009-4016 (2015).

${ }^{47}$ M. D. LaCount and M. T. Lusk, "Improved energy pooling efficiency through inhibited spontaneous emission," J. Phys. Chem. C 121, 8335-8344 (2017).

${ }^{48} \mathrm{X}$. Zhong, T. Chervy, L. Zhang, A. Thomas, J. George, C. Genet, J. A. Hutchison, and T. W. Ebbesen, "Energy transfer between spatially separated entangled molecules," Angew. Chem., Int. Ed. 56, 9034-9038 (2017).

${ }^{49}$ R. D. Jenkins and D. L. Andrews, "Four-centre energy transfer and interaction pairs: Molecular quantum electrodynamics," J. Chem. Phys. 116, 6713-6724 (2002).

${ }^{50} \mathrm{~J}$. R. Zurita-Sánchez and J. Méndez-Villanueva, "Förster Energy transfer in the vicinity of two metallic nanospheres (dimer)," Plasmonics 13, 873-883 (2018).

${ }^{51}$ A. Salam, "Near-zone mediation of RET by one and two proximal particles," J. Phys. Chem. A 123, 2853-2860 (2019).

${ }^{52}$ A. Salam, "Mediation of resonance energy transfer by two polarisable particles," J. Chem. Phys. 151, 244119 (2019).

${ }^{53}$ G. S. Engel, T. R. Calhoun, E. L. Read, T.-K. Ahn, T. Mančal, Y.-C. Cheng, R. E. Blankenship, and G. R. Fleming, "Evidence for wavelike energy transfer through quantum coherence in photosynthetic systems," Nature 446, 782-786 (2007).

${ }^{54}$ E. Collini, C. Y. Wong, K. E. Wilk, P. M. G. Curmi, P. Brumer, and G. D. Scholes, "Coherently wired light-harvesting in photosynthetic marine algae at ambient temperature," Nature 463, 644-647 (2010).

${ }^{55}$ G. Panitchayangkoon, D. Hayes, K. A. Fransted, J. R. Caram, E. Harel, J. Wen, R. E. Blankenship, and G. S. Engel, "Long-lived quantum coherence in photosynthetic complexes at physiological temperature," Proc. Natl. Acad. Sci. U. S. A. 107, 12766-12770 (2010).

${ }^{56} \mathrm{~T}$. Mančal, "A decade with quantum coherence: How our past became classical and the future turned quantum," Chem. Phys. 532, 110663 (2020).

${ }^{57}$ H. Mabuchi and A. C. Doherty, "Cavity quantum electrodynamics: Coherence in context," Science 298, 1372-1377 (2002).

${ }^{58}$ Y.-W. Lu, L.-Y. Li, C.-L. You, and J.-F. Liu, "Spectra of plasmon-exciton composite under weak coherent pumping within cavity QED treatment," J. Phys. B: At., Mol. Opt. Phys. 53, 035401 (2020).

${ }^{59}$ D. L. Andrews and K. A. Forbes, "Quantum features in the orthogonality of optical modes for structured and plane-wave light," Opt. Lett. 43, 3249-3252 (2018).

${ }^{60}$ H. Hossein-Nejad, A. Olaya-Castro, and G. D. Scholes, "Phonon-mediated path-interference in electronic energy transfer," J. Chem. Phys. 136, 024112 (2012).

${ }^{61}$ G. C. Solomon, D. Q. Andrews, T. Hansen, R. H. Goldsmith, M. R. Wasielewski, R. P. Van Duyne, and M. A. Ratner, "Understanding quantum interference in coherent molecular conduction," J. Chem. Phys. 129, 054701 (2008).

${ }^{62}$ N. Darwish, I. Díez-Pérez, P. Da Silva, N. Tao, J. J. Gooding, and M. N. PaddonRow, "Observation of electrochemically controlled quantum interference in a single anthraquinone-based norbornylogous bridge molecule," Angew. Chem., Int. Ed. 51, 3203-3206 (2012).

${ }^{63}$ D. L. Andrews and T. Thirunamachandran, "On three-dimensional rotational averages,” J. Chem. Phys. 67, 5026-5033 (1977).

${ }^{64} \mathrm{H}$. Yabuki, "Feynman path integrals in the Young double-slit experiment," Int. J. Theor. Phys. 25, 159-174 (1986). 